\title{
Revision of the Orbamia Herbulot, 1966 group of genera with description of two new genera, ten new species, and two new subspecies (Lepidoptera, Geometridae, Ennominae, Cassymini)
}

\author{
Tesfu Fekensa Tujuba ${ }^{1,3}$, Axel Hausmann², Andrea Sciarretta ${ }^{3}$ \\ I Ethiopian Biodiversity Institute, Comoros street, Addis Ababa, Ethiopia 2 SNSB-Zoologische Staatssa- \\ mmlung München, Münchhausenstr, 21, Munich, Germany 3 Department of Agriculture, Environment and \\ Food Sciences, University of Molise, Via Francesco De Sanctis Campobasso, Italy \\ Corresponding author: Axel Hausmann (hausmann.a@snsb.de)
}

Academic editor: E.J. van Nieukerken | Received 22 January 2020 | Accepted 16 March 2020 | Published 22 April 2020

http://zoobank.org/D5E1D8E5-7DF3-4408-93ED-94CDE2C59FEA

Citation: Tujuba TF, Hausmann A, Sciarretta A (2020) Revision of the Orbamia Herbulot, 1966 group of genera with description of two new genera, ten new species, and two new subspecies (Lepidoptera, Geometridae, Ennominae, Cassymini). ZooKeys 929: 53-77. https://doi.org/10.3897/zookeys.929.50391

\begin{abstract}
The genus Orbamia Herbulot, 1966 is revised. Two new genera are described: Rabomia Hausmann \& Tujuba, gen. nov. (type species: Ectropis? subaurata Warren, 1899), and Morabia Hausmann \& Tujuba, gen. nov. (type species: Morabia politzari Hausmann \& Tujuba, sp. nov.). Ten new species and two new subspecies are described: Rabomia obscurior Hausmann \& Tujuba, sp. nov., from western Africa, Morabia politzari Hausmann \& Tujuba, sp. nov., from Kenya, Morabia brunnea Hausmann \& Tujuba, sp. nov., from Zambia, Orbamia marginata Hausmann \& Tujuba, sp. nov., from Tanzania, Orbamia clarissima Hausmann \& Tujuba, sp. nov., from Kenya, Orbamia clarior Hausmann \& Tujuba, sp. nov., from Kenya, Orbamia obliqua Hausmann \& Tujuba, sp. nov., from Zambia, Orbamia obliqua parva Hausmann \& Tujuba, subsp. nov., from South Africa, Orbamia abiyi Hausmann \& Tujuba, sp. nov., from Zambia, Tanzania, Ethiopia, Orbamia emanai Hausmann \& Tujuba, sp. nov., from Ethiopia, Orbamia emanai lenzi Hausmann \& Tujuba, subsp. nov., from Zambia and Malawi, and Orbamia balensis Hausmann \& Tujuba, sp. nov. from Ethiopia. The taxon Lepiodes ocellata Warren, 1897 is raised from synonymy of $O$. octomaculata (Wallengren, 1872) to species rank (Zambia, Tanzania, Rwanda). The taxonomical analysis is based on both morphological and genetic cytochrome oxidase I (COI) data. Adults and male and female genitalia of all species are illustrated.
\end{abstract}

Copyright Tesfu Fekensa Tujuba et al. This is an open access article distributed under the terms of the Creative Commons Attribution License (CC BY 4.0), which permits unrestricted use, distribution, and reproduction in any medium, provided the original author and source are credited. 


\section{Keywords}

Africa, DNA Barcoding, geometrid moths, integrative taxonomy, Lepidoptera, Morabia, Rabomia, species description

\section{Introduction}

After 260 years of intensive work, taxonomists worldwide have together achieved the formal descriptions of approximately 160,000 lepidopteran species (Van Nieukerken et al. 2011), of which some 24,000 are geometrid moths (cf. Scoble 1999; Scoble and Hausmann 2007; Van Nieukerken et al. 2011). Many more are likely to be described and some recent revisions of many genera revealed high percentages of undescribed species (e.g., Brehm 2015, 2018; Hausmann 2003; Hausmann et al. 2016). This probably covers less than half the number of actual extant geometrid species on earth, an estimate which can be inferred from apparently undescribed species in natural history museums and in molecular databases like the Barcode of Life Data Systems (BOLD: Ratnasingham and Hebert 2007). We conclude that conventional taxonomy with an actual description rate of 80-100 species per year works too slowly for addressing the biodiversity of our Earth (taxonomic impediment: de Carvalho et al. 2007; Wheeler 2008). In these times where we are facing a serious extinction rate we cannot afford to wait for another 250-600 years for the taxonomic assessment of our biodiversity. In the recent literature, several pleas for an accelerated taxonomy have been made (Riedel et al. 2013a; Forum Herbulot 2014) and some taxonomists already published exemplary revisions with shortened descriptions (Riedel et al. 2013b; Meierotto et al. 2019).

In this taxonomic revision we follow such a model of accelerated taxonomy (cf. Riedel et al. 2013a, 2013b; Forum Herbulot 2014; Meierotto et al. 2019), which should lead in future to an automated, easy and rapid transfer of genetic data, images and metadata directly from BOLD into manuscripts and which will allow for continuous updates. Similarly, all nomenclatorial information (valid names, synonyms, original descriptions with authorship and year, type localities, type specimens and their deposition) may in future be transferred in an automated way either from BOLD, from the Global Lepidoptera checklist (Banki et al. 2019) and/or from the Geometridae Mundi database once it will be completed by the Forum Herbulot initiative (cf. Löbel and Hausmann 2019). Similar to the approach of Meierotto et al. (2019) we believe that DNA barcodes in most cases are an excellent tool for species diagnosis but that all descriptions should be linked with a brief description of characters in words, supplemented with photographs. In future, such revisions may also be organised in a flexible way, i.e., with the possibility to subsequently publish updated versions (keeping the previous versions visible) with more data, newly added species, and revised taxonomic concepts. In the framework of the ongoing research project "GBOL III - Dark Taxa" (SNSB - Bavarian State Collection of Zoology, Munich) similar workflows are planned to be tested and established.

The genus Orbamia Herbulot, 1966 is restricted to the Ethiopian region, where it was so far represented by five species (Scoble 1999; Hausmann 2006): Orbamia 
octomaculata (Wallengren 1872), Orbamia pauperata Herbulot, 1966, Orbamia renimacula (Prout, 1926), Orbamia subaurata (Warren, 1899) and Orbamia becki Hausmann, 2006. Although the external appearance of Orbamia is somewhat reminiscent of that of the tribe Boarmiini, Hausmann (2006) placed Orbamia in the tribe Cassymini, due to the long process extending from the base of the dorsal margin of the male genital valvae, similar to the equivalent present in Zamarada. Molecular evidence for this tribal assignment was given by Murillo-Ramos et al. (2019) and Brehm et al. (2019). Species of Orbamia are recognised by the following characters: wings with conspicuous, contrasting discal spots on all wings, antennae bipectinate in males and filiform in females, dorsal process in male genitalia strongly curved.

\section{Materials and methods}

In the present paper, the material housed in the Zoologische Staatssammlung München (ZSM), Munich, Germany, collected from 22 different African and Arabian countries, has been studied. Two relevant type specimens available in the ZSM and two from the Natural History Museum in London (NHMUK) were examined. Altogether, 298 specimens of the genera Orbamia, Rabomia gen. nov., and Morabia gen. nov. have been examined.

Comparative morphological methods and COI sequence divergences were used to delimit the taxa and to estimate their taxonomic status. We studied morphological characters of adults (including genitalia and wing venation). The abdomens and genitalia were prepared using the method of Hardwick (1950). The analysis is furthermore based on 58 genitalia slides and 72 DNA barcodes.

For DNA analyses, one or two legs were removed from dried specimens and stored in an individual tube, in absolute ethanol. DNA extraction, amplification and sequencing of the "barcode" region of the mitochondrial cytochrome c oxidase I (COI) gene region (658 base pairs) were carried out in the Canadian Centre for DNA Barcoding, Ontario, Canada, using standard high through-put protocols (Ivanova et al. 2006). Sequence divergences within and between species were calculated using the Kimura 2-parameter model (Kimura 1980), using the analytical tools provided by BOLD Systems v4 platform (Ratnasingham and Hebert 2007; http://www.boldsystems.org/). Intra-specific and inter-specific genetic distances are reported as maximum and minimum distances, respectively. The Barcode Index Number (BIN) of each species is reported which was obtained from the BOLD Systems v4 database. BINs represent a species-level taxonomic registry of the animal kingdom based on the analysis of nucleotide variation patterns in the barcode region of the cytochrome c oxidase I (COI) gene (Ratnasingham and Hebert 2013). This genetic information facilitates the species delimitation and constitutes the basis of future phylogenetic works at the genus level and below (Brehm 2015, 2018).

Label data and photographs of types and other barcoded specimens are accessible on BOLD, dataset DS-ORBAMIA (https:/dx.doi.org/10.5883/DS-ORBAMIA). All new names are registered in ZooBank. Geo-references were taken from specimen labels. 


\section{Systematic accounts}

\section{Orbamia Herbulot, 1966}

Herbulot (1966): 221. Type species: Orbamia octomaculata Wallengren, 1872

Differential features (COI sequences, photographs of adults and their genitalia see https://dx.doi.org/10.5883/DS-ORBAMIA). Adult: Male antennae bipectinate. Upperside of wings with conspicuous, contrasting discal spots on all wings. Underside with yellowish scales, with darker pattern towards termen. Male genitalia: Differing from those of the other two genera by longer uncus, naked dorsal process of valva, long and narrow cornutus. Female genitalia: Apophyses anteriores usually half-length of apophyses posteriores, lamellae ante- and post-vaginalis sclerotised (often oval), ductus bursae membranous, signum a small sclerite with transverse ridge.

Genetic data and phylogeny. Multigene analyses of Geometridae revealed evidence for assignation of the genus Orbamia to Cassymini and for sister group relationship with the African genus Pycnostega (Murillo-Ramos et al. 2019; Brehm et al. 2019). The maximum likelihood analysis of COI barcode data underpins the monophyly of the genus Orbamia as conceived and circumscribed here (cf. Table 1, Fig. 85).

\section{Orbamia octomaculata (Wallengren, 1872)}

BIN: BOLD: AAQ4039

Figures 1, 19, 37, 55, 73

Panagra octomaculata: Wallengren (1872): 60 (Holotype ô in NHRS, Stockholm; locus typicus: [South Africa]: "Caffraria orientalis interior")

Examined material (ZSM). 25 $\hat{\jmath}$ 우 from Tanzania, Zambia, Botswana, Mozambique, Malawi, Namibia, South Africa (ZSM G 20929/क; ZSM G 20921/

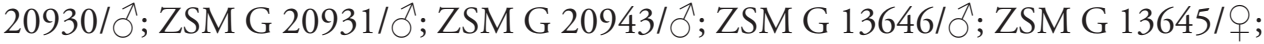
ZSM G 20947/P).

Differential features (COI sequences, photographs of adults and their genitalia see https://dx.doi.org/10.5883/DS-ORBAMIA): Adult: Forewing length: 11.5$13.5 \mathrm{~mm}$. Upperside of wings: Ground colour pale brown with dark suffusion. Underside: Ground colour yellow or orange with much dark suffusion, terminal fascia on hind wing usually complete, on forewing restricted to apex. Male genitalia: Uncus long, triangular, valva strongly (rectangularly) bent, dorsal process with small spinule at tip, cornutus short and stout $(1.4-1.7 \mathrm{~mm})$. Female genitalia: Lamella antevaginalis U-shaped, long $(0.75 \mathrm{~mm})$, signum sclerotised, transversely flat, transverse ridge curved $(0.4-0.5 \mathrm{~mm})$. 
Table I. Barcode gap analysis of COI data of the genera Orbamia, Rabomia, and Morabia (https://dx.doi. org/10.5883/DS-ORBAMIA), showing intraspecific variation ("Mean Intra-Sp" and "Max. Intra-Sp.") and distances from Nearest Neighbour Species ("NN").

\begin{tabular}{lcccc}
\hline \multicolumn{1}{c}{ Species } & Mean Intra-Sp & Max. Intra-Sp & Nearest species & Distance NN \\
\hline Orbamia abiyi & 0.51 & 0.93 & Orbamia clarior & 2.1 \\
Orbamia balensis & 0.61 & 0.61 & Orbamia ocellata & 7.65 \\
Orbamia becki & 0.97 & 2.68 & Orbamia marginata & 1.89 \\
Orbamia clarior & 0 & 0 & Orbamia abiyi & 2.1 \\
Orbamia ocellata & 0 & 0 & Orbamia clarior & 2.81 \\
Orbamia emanai & 1.43 & 2.17 & Orbamia clarior & 2.47 \\
Orbamia marginata & 0.53 & 0.62 & Orbamia becki & 1.89 \\
Orbamia obliqua & 1.35 & 1.87 & Orbamia abiyi & 3.63 \\
Orbamia octomaculata & 0.69 & 1.27 & Orbamia marginata & 2.34 \\
Orbamia renimacula & 0.42 & 1.08 & Orbamia becki & 2.18 \\
Rabomia obscurata & N/A & 0 & Rabomia subaurata & 5.19 \\
Rabomia subaurata & 0.41 & 0.62 & Rabomia obscurata & 5.19 \\
Morabia brunnea & N/A & 0 & Morabia politzari & 2.66 \\
Morabia politzari & 0.25 & 0.26 & Morabia brunnea & 2.66 \\
\hline
\end{tabular}

\section{Orbamia marginata Hausmann \& Tujuba, sp. nov.}

http://zoobank.org/6B79EAB5-4CE5-4B46-9176-5A47FF51E0AB

BIN: BOLD: AAZ5266

Figures 2, 20, 38, 56

Examined material. Holotype: $\curvearrowright$, Tanzania, Bukwa region, $14 \mathrm{~km}$ W Namanyere, 1290 m, 07²7.28'S, 03054.49'E, 14.xi.2005, leg. Ph. Darge, coll. ZSM (ZSM G 20909).

Paratypes: 2ð̄, Tanzania, Rukwa region, $14 \mathrm{~km}$ W Namanyere, $1290 \mathrm{~m}$,

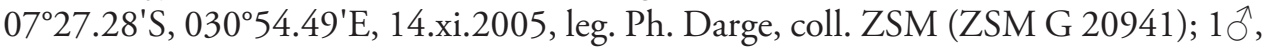
Tanzania, Rukwa region, Luafi Game Reserve (W. Namanyere), 1260 m, 07²6.98'S, $030^{\circ} 54.24^{\prime} \mathrm{E}, 31.1 .2008$, leg. Ph. Darge; 10̄, Tanzanie, Iringa region, Iyayi savanna, $1400 \mathrm{~m}, 08^{\circ} 51.47^{\prime} \mathrm{S}, 034^{\circ} 31.29^{\prime} \mathrm{E}, 14 . i v .2007, \mathrm{Ph}$. Darge; $1 \mathrm{O}^{\Uparrow}$, Tanzania, Morogoro region, Udzungwa N.P. camp site, $3315 \mathrm{~m}, 07^{\circ} 50.95^{\prime} \mathrm{S}, 036^{\circ} 50.95^{\prime} \mathrm{E}, 26 . x \mathrm{x} .2005$, leg. Ph. Darge (all ZSM).

Etymology. The name refers to the uninterrupted black line at the hindwing margin (Lat. margo/marginis = edge, border).

Differential features (COI sequences, photographs of adults and their genitalia see https://dx.doi.org/10.5883/DS-ORBAMIA): Adult: Forewing length: 11.5-12.5 mm. Upper side of wings: Ground colour dirty grey with brown suffusion. Underside: Ground colour whitish beige with some dark suffusion, terminal fascia conspicuous, uninterrupted on all wings. Male genitalia: Uncus long, triangular, valva slightly bent, dorsal process with small spinule at tip, cornutus short and stout $(1.6 \mathrm{~mm})$. Female genitalia unknown. 


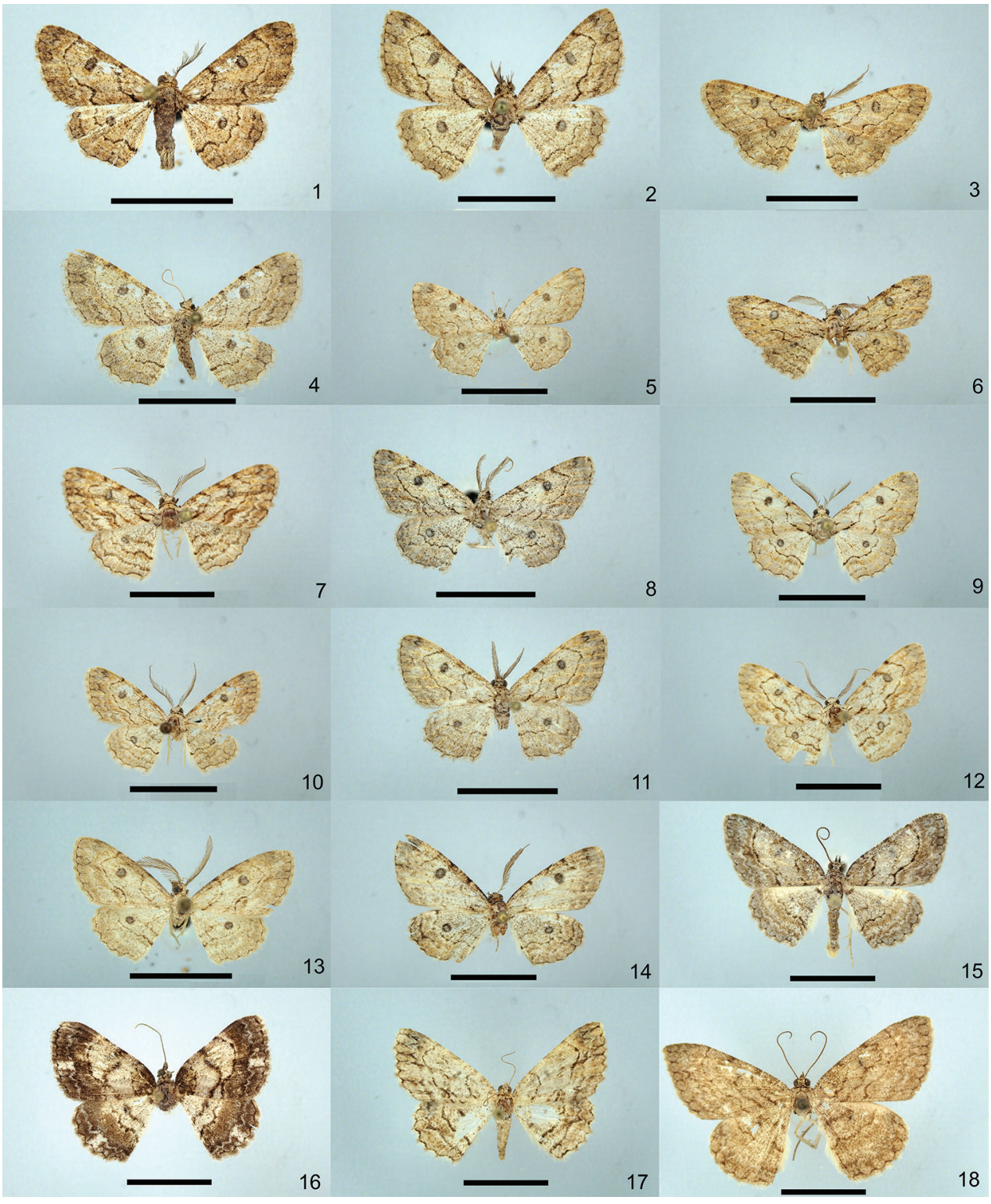

Figures I-18. Specimens of the genera Orbamia, Rabomia, and Morabia, dorsal view. I Orbamia octomaculata 2 O. marginata (paratype) 3 O. becki (holotype) 4 O. renimacula 5 O. clarissima (holotype) 6 O. clarior (holotype) 7 O. obliqua (holotype) 8 O. obliqua parva (holotype) 9 O. ocellata 10 O. abiyi (holotype) II O. emanai (holotype) I2 O. emanai lenzi (holotype) I3 O. pauperata (holotype) I4 O. balensis (holotype) I5 Rabomia subaurata 16 R. obscurior (holotype) 17 Morabia politzari (holotype) 18 M. brunnea (holotype). Scale bars: $1 \mathrm{~cm}$. 


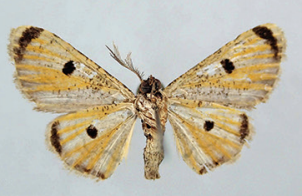

19

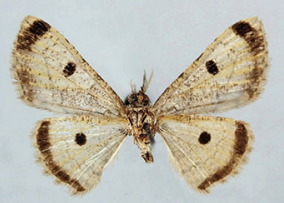

20

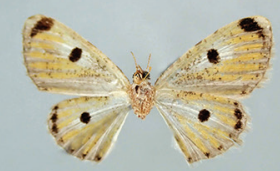

23

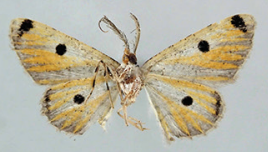

25

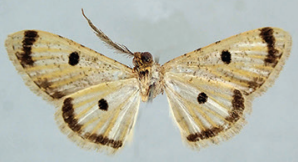

21

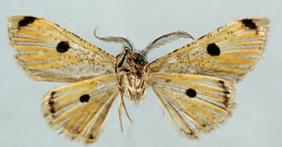

24

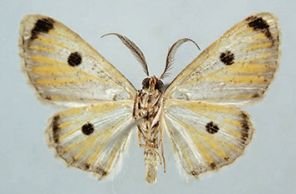

27

Figures 19-27. Specimens of the genus Orbamia, ventral view. 19 Orbamia octomaculata 20 O. marginata $\mathbf{2 1}$ O. becki $\mathbf{2 2}$ O. renimacula $\mathbf{2 3}$ O. clarissima $\mathbf{2 4}$ O. clarior $\mathbf{2 5}$ O. obliqua $\mathbf{2 6}$ O. obliqua parva 27 O. ocellata. Scale bars: $1 \mathrm{~cm}$.

\section{Orbamia becki Hausmann, 2006}

BIN: BOLD: AAD8768

Figures 3, 21, 39, 57, 74

Orbamia becki: Hausmann (2006): 42 (Holotype ${ }^{\lambda}$ in ZSM: G 13627; locus typicus: Yemen: Al Hudaydah, Jebel Burra).

Examined material (ZSM). 57ð⿱ 9 from Yemen, Ethiopia and Djibouti (ZSM G

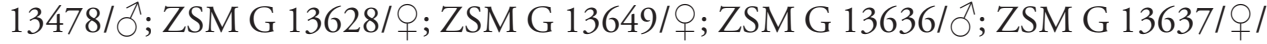
ZSM G 13650/ㅇ; ZSM G 20927/ㅇ).

Differential features (COI sequences, photographs of adults and their genitalia see https://dx.doi.org/10.5883/DS-ORBAMIA): Adult: Forewing length: 9.5$12 \mathrm{~mm}$. Upperside of wings: Ground colour pale brown. Underside: Ground colour whitish with yellowish tinge, on forewing with some dark suffusion, terminal fascia on all wings complete. Male genitalia very similar to those of $O$. octomaculata: Uncus stout, triangular, valva strongly (rectangularly) bent, dorsal process with small spinule at tip, cornutus short and stout $(1.5 \mathrm{~mm})$. Female genitalia very similar to those of 
O. octomaculata: Lamella antevaginalis U-shaped $(0.6-0.7 \mathrm{~mm})$, signum sclerotised, transversely oval, transverse ridge curved $(0.3-0.5 \mathrm{~mm})$.

Remarks. Allopatric vicariant of O. octomaculata. Orbamia becki is the only species of this genus that has also been recorded outside Africa in Yemen, southern Arabia.

\section{Orbamia renimacula (Prout, 1926)}

BIN: BOLD: AAE1536

Figures 4, 22, 40, 58, 75

Boarmia renimacula: Prout (1926): 184 (holotype 9 in NHMUK; locus typicus: Senegal: Sédhiou).

Examined material (ZSM). $45 \overbrace{}^{\lambda}$ ㅇ from Cameroon, Burkina Faso (Upper Volta),

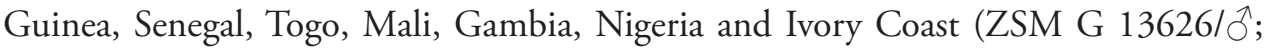
ZSM G 61321/क; ZSM G 13620/ぶ).

Differential features (COI sequences, photographs of adults and their genitalia see https://dx.doi.org/10.5883/DS-ORBAMIA): Adult: Forewing length: 8.5-12 mm, one of the smallest Orbamia species. Upperside of wings: Ground colour pale grey with brown suffusion. Underside: Ground colour white with very slight yellowish tinge, and slight dark suffusion, terminal fascia on all wings complete, conspicuous, at forewing apex dilated. Male genitalia: Uncus narrow digitiform, saccus round, valva almost straight, dorsal process with fine, curved hook at tip, cornutus of medium length (2.0 $\mathrm{mm})$. Female genitalia: Lamella post-vaginalis weakly sclerotised, lamella ante-vaginalis U-shaped $(0.6 \mathrm{~mm})$, signum sclerotised, rhomboid, transverse ridge straight $(0.25 \mathrm{~mm})$.

\section{Orbamia clarissima Hausmann \& Tujuba, sp. nov.}

http://zoobank.org/38E93700-3258-4497-B742-39B80BB75B0D

BIN: not yet assigned, DNA barcodes BC ZSM Lep 106553, 106554, 106555.

Figures 5, 23, 41, 59, 76

Examined material. Holotype: + , Kibwezi, B.E.A. [Kenya], 12 March 1917 (W. Feather), coll. ZSM (ZSM G 13618).

Paratypes: $1{ }^{\lambda}$, Kibwezi, B.E.A. [Kenya], April 1922 (W. Feather); $10^{\Uparrow}$, Kenya, Mutha, 5.IV.69, Watulege; 19, Kenya, Musthomo, 13.III.69, Watulege (ZSM G 13617); 10ิ, Somalia m., Caonole Fluß, 21.1.1988, leg. Dr. Politzar (all ZSM). ZSM G 13647/ठ̂.

Etymology. The name refers to the very pale ground colour (Lat. clarissimus, -a, -um = palest, clearest).

Differential features (COI sequences, photographs of adults and their genitalia see https://dx.doi.org/10.5883/DS-ORBAMIA): Adult: Forewing length: 7.5-11 mm, one of the smallest Orbamia species. Upperside of wings: Ground colour whitish with slight grey brown suffusion. Underside: Ground colour white with slight orange tinge, 
mainly towards termen between veins, apical spots on all wings conspicuous, sharp. Male genitalia: Uncus long, digitiform, stout, valva straight, dorsal process with small spinule at tip, cornutus very short $(1.0 \mathrm{~mm})$ and tiny. Female genitalia: Apophyses stout, apophyses anteriores comparatively long (2/3 length of apophyses posteriores), lamella ante-vaginalis heart-shaped (length and width $0.45 \mathrm{~mm}$ ), signum small, sclerotised, transverse ridge straight $(0.15 \mathrm{~mm})$.

\section{Orbamia clarior Hausmann \& Tujuba, sp. nov.}

http://zoobank.org/79B39635-880A-411F-B9C8-563320CA5766

BIN: BOLD: ABW5825

Figures 6, 24, 42, 60

Examined material. Holotype: Ô, Kenya, South Ukanbasi, 6.V.1996, leg. Politzar (ZSM G 20944).

Etymology. The name refers to the comparatively pale ground colour.

Differential features (COI sequences, photographs of adults and their genitalia see https://dx.doi.org/10.5883/DS-ORBAMIA): Forewing length: $10 \mathrm{~mm}$. Upperside of wings: Ground colour comparatively dark brownish, transverse lines of forewing oblique. Underside: Ground colour whitish beige, orange between veins, apical spot on forewing conspicuous, sharp, on hindwing narrow, elongate, remnants of dark colouration in the anal angle. Male genitalia: Uncus of medium length, digitiform, stout, dilated towards base, valva straight, dorsal process with conspicuous spinule at tip, cornutus very long $(2.7 \mathrm{~mm})$. Female genitalia unknown.

\section{Orbamia obliqua Hausmann \& Tujuba, sp. nov.}

http://zoobank.org/BED6B29E-D967-419C-A998-6773D7BF078D

BIN: BOLD: AAM4892

Figures 7, 25, 43, 61

Examined material. Holotype: ${ }^{\lambda}$, North-western Zambia, Hillwood farm, $11^{\circ} 16.01^{\prime} \mathrm{S}$, 24ํ⒙99'E, 17.ix.2009, 1420 m, UV, J. Lenz legit, coll. ZSM (G 20905).

Paratype: $1 \hat{0}$, Tanzania, Morogoro province, Nguru mounts, IV.2004 (ex coll. Philippe Darge, coll. ZSM, G 20906).

Etymology. The name refers to the oblique position of the transverse lines of the forewing (Lat. obliquus, $-\mathrm{a}$, -um = oblique).

Differential features (COI sequences, photographs of adults and their genitalia see https://dx.doi.org/10.5883/DS-ORBAMIA): Adult: Forewing length: 11-12 mm. Upperside of wings: Ground colour pale grey with brown pattern, transverse lines oblique. Underside: Ground colour beige with slight yellowish tinge, and with strong dark suffusion, terminal area with pattern reduced to a dark apical spot and a dark shadow on the hindwing apex. Male genitalia: Uncus short, stout, hook-shaped, saccus 
projection shallow, valva straight, narrow at tip, dorsal process with a stout hook at tip, cornutus of medium length $(1.9 \mathrm{~mm})$. Female genitalia unknown.

\section{Orbamia obliqua parva Hausmann \& Tujuba, subsp. nov.}

http://zoobank.org/3A0A5166-35FB-4D67-9BC2-B4C5C1024779

BIN: BOLD: AAM4892

Figures 8, 26, 44, 62

Examined material. Holotype: $\widehat{\jmath}$, South Africa, Limpopo, Melkrivier Lapalala, Wilderness Kolobe camp, 1220 m -23.9094/28.2736, 13.xi.2017, leg. A. Hausmann, coll. Ditsong Museum, Pretoria, gen. prp. ZSM G 20933.

Paratypes: $6{ }^{\lambda}$, South Africa, Limpopo, Melkrivier Lapalala, Wilderness, Kolobe camp, 1220 m -23.9094/28.2736, 13.xi.2017, leg. A. Hausmann; 2ð, RSA, North-

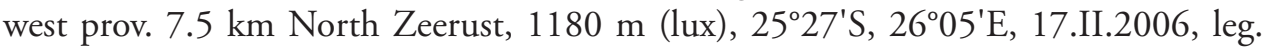
Hacker (ZSM G 20949); $1 \jmath^{\Uparrow}$, South Africa, Gauteng, Mogale’s Gate Biodiversity Centre, near Bush Camp, -25.938, 27.639, 1420 m, 14.ii.2012, leg. P. Hebert, J. deWaard, coll. University of Guelph (Canada), Centre for Biodiversity Genomics.

Etymology. The name refers to the small size of this subspecies (Lat. parvus, -a, -um = small), being much smaller than the sympatric $O$. octomaculata.

Differential features (COI sequences, photographs of adults and their genitalia see https://dx.doi.org/10.5883/DS-ORBAMIA): Adult: Forewing length: 9-11 mm. Upperside of wings: Ground colour pale grey, darker in the terminal area, pattern dark grey. Underside: Ground colour whitish beige, orange between veins, on forewing apex a sharp black spot, dark colouration on the hindwing terminal area restricted to a small stripe or shadow in the apex. Male genitalia: Uncus comparatively short, stout, hookshaped, saccus projection shallow, valva straight, narrow at tip, dorsal process with a stout hook at tip, cornutus of medium length $(1.6-1.9 \mathrm{~mm})$. Female genitalia unknown.

\section{Orbamia ocellata (Warren, 1897), stat. nov.}

BIN: BOLD: AAP8312

Figures 9, 27, 45, 63, 77

Lepiodes ocellata: Warren (1897): 94 (Syntypes 5つ1 in NHMUK; locus typicus: South Africa: Bathurst; [north-eastern Zambia]: Mpeta, Loangwa River [Luangwa], off the Zambesi [close to the border with northern Malawi]).

Note. Synonym of O. octomaculata according to Scoble (1999) but the type series of ocellata belongs to two different species. Herewith we designate the red-ring-labelled male specimen from Zambia, Mpeta, as lectotype to fix the identity of the name and to stabilise nomenclature. The taxon is herewith upgraded from synonymy to species rank (stat. nov.), based on the below mentioned differences in DNA barcodes and genitalia. Pattern of upper- and underside of wings of the lectotype exactly matches that of the 
examined material from Tanzania, partly collected in closely adjacent localities to the type locality (e.g., Ruvuma and Iringa provinces).

Examined material. $1 \delta^{\top}$, Tanzania, Pwani region, Manadera, $166 \mathrm{~m}, 06^{\circ} 14.30^{\prime} \mathrm{S}$, 038²3.19'E, 07.XII.2008, leg. Ph. Darge (ZSM G 20908); 1 ㅇ, Tanzanie, Tanga region, Savane près, Usambara west. 475 m, 11.v.2005 leg. Ph. Darge; 2 q, Tanzanie, Tanga region, West Usambara mts, Magamba Forest, 1818 m, 04²42.76'S, 038 17.28'E, 01.XII.2008, leg. Ph. Darge; 19, Tanzanie, Morongoi region, Mikesse Hills, 375 m, 06 40.50'S, 03758.12'E, 17.XI.2004, leg. Ph. Darge; 2q, Tanzanie, Morogoro 1 km E Mikumi, 550 m, 5.III.2003, leg. M. Fibiger, H. Hacker, K.Larsen, H.P. Schreier; 1 q, Tanzania, Morogoro region, Uluguru mts, Bunduki Forest, 1275 m, 0701.07'S, 037³7.94'E, 23.XI.2007, leg. Ph. Darge; 19 , Tanzania, Morogoro region, face West des, Nguru mts, Makuyu, savane arboree, 620 m, 25.IV.2005, leg. Ph. Darge (ZSM G 20942); 29, Tanzania, Morogoro region, West Nguru mts, Makuyu, alt. 620 m, 06²16.08'S, 037²0.54'E, 19.XI.2007, leg. Ph. Darge; $1 \delta^{\top}$, Tanzania, Rukwa prov, Kisengere/ Kasambo, 1193 m, 07²7.54'S, 030 $52.81^{\prime} \mathrm{E}$, 17.v.2004, leg. Ph. Darge; $1 \delta^{\lambda}$, Tanzania, Rukwa prov., Mbizi mts, entre Kisungu et Muze, $1415 \mathrm{~m}, 07^{\circ} 43.82^{\prime} \mathrm{S}, 031^{\circ} 32.4^{\prime} \mathrm{E}, 14 . v .2004$, leg. Ph. Darge; 1 q, Tanzanie, Iringa, Ulembwa, $2070 \mathrm{~m}, 09^{\circ} 18.70^{\prime} \mathrm{S}, 034^{\circ} 38.07^{\prime} \mathrm{E}, 22 . X I I .2008$, leg. Ph. Darge (ZSM G 20911); 2 ㅇ, Tanzanie, Pwani region, Savane de Mandera, 170 m, 06 ${ }^{\circ} 14.30^{\prime} \mathrm{S}, 038^{\circ} 23.19^{\prime} \mathrm{E}, 19.1 \mathrm{II} .2006$, leg. Ph. Darge; $1 \delta^{\top} 2$, id., 15.I.2005; 1․ Tanzanie, Ruvuma region, Kitai Savnna, 1020 m, 1042.40'S, 035¹2.33'E, 24.III.2006, leg. Ph. Darge (ZSM G 20946); 10, Tanzanie, Mbeya region, Igurusi savanna, $1150 \mathrm{~m}, 08^{\circ} 46.68^{\prime} \mathrm{S}, 033^{\circ} 46.17^{\prime} \mathrm{E}, 06 . I V .2006$, leg. Ph. Darge; 39, Tanzania, Ubenazomizi region, savannas and deciduous forest, $450 \mathrm{~m}$, 06 40.57'S, 03758.99'E, 13.XII.2002, leg. Ph. Darge and Th. Ebode; 1 ㅇ, Tanzanie, Ruvuma region, Kitai Savanna, 1020 m, 1042.40'S, 035¹2.33'E, 24.III.2006, leg. Ph. Darge.

Differential features (COI sequences, photographs of adults and their genitalia see https://dx.doi.org/10.5883/DS-ORBAMIA): Adult: Forewing length: 8.5-12 mm. Upperside of wings: Ground colour pale grey with slight brown suffusion, pattern brown with slight orange tinge. Underside: Ground colour whitish, orange between veins, apical spots on forewing conspicuous, sharply bordered, on hindwing terminal fascia usually diffuse, rarely restricted to apex. Male genitalia: Uncus narrow, digitiform, valva straight, broad, dorsal process with conspicuous, stout hook at tip, cornutus narrow and very long $(2.8-3.0 \mathrm{~mm})$. Female genitalia: Apophyses stout, apophyses anteriores comparatively long (2/3 length of apophyses posteriores), lamella ante- and post-vaginalis fused, oval, comparatively broad (length $0.7 \mathrm{~mm}$, width $0.5 \mathrm{~mm}$ ), signum weakly sclerotised, small, transverse ridge straight $(0.2 \mathrm{~mm})$.

\section{Orbamia abiyi Hausmann \& Tujuba, sp. nov.}

http://zoobank.org/8AC03FE5-C57D-4BA1-9C63-44281F0AB521

BIN: BOLD: AAK5536

Figures 10, 28, 46, 64, 78

Examined material. Holotype: $\widehat{\jmath}$, North Zambia, Mutinondo, 1390 m, wet Miombo, 29.XII. 2010, 12²3'30"S, 31 19'23"E, light trap, J. Lenz legit (ZSM G 20912). 


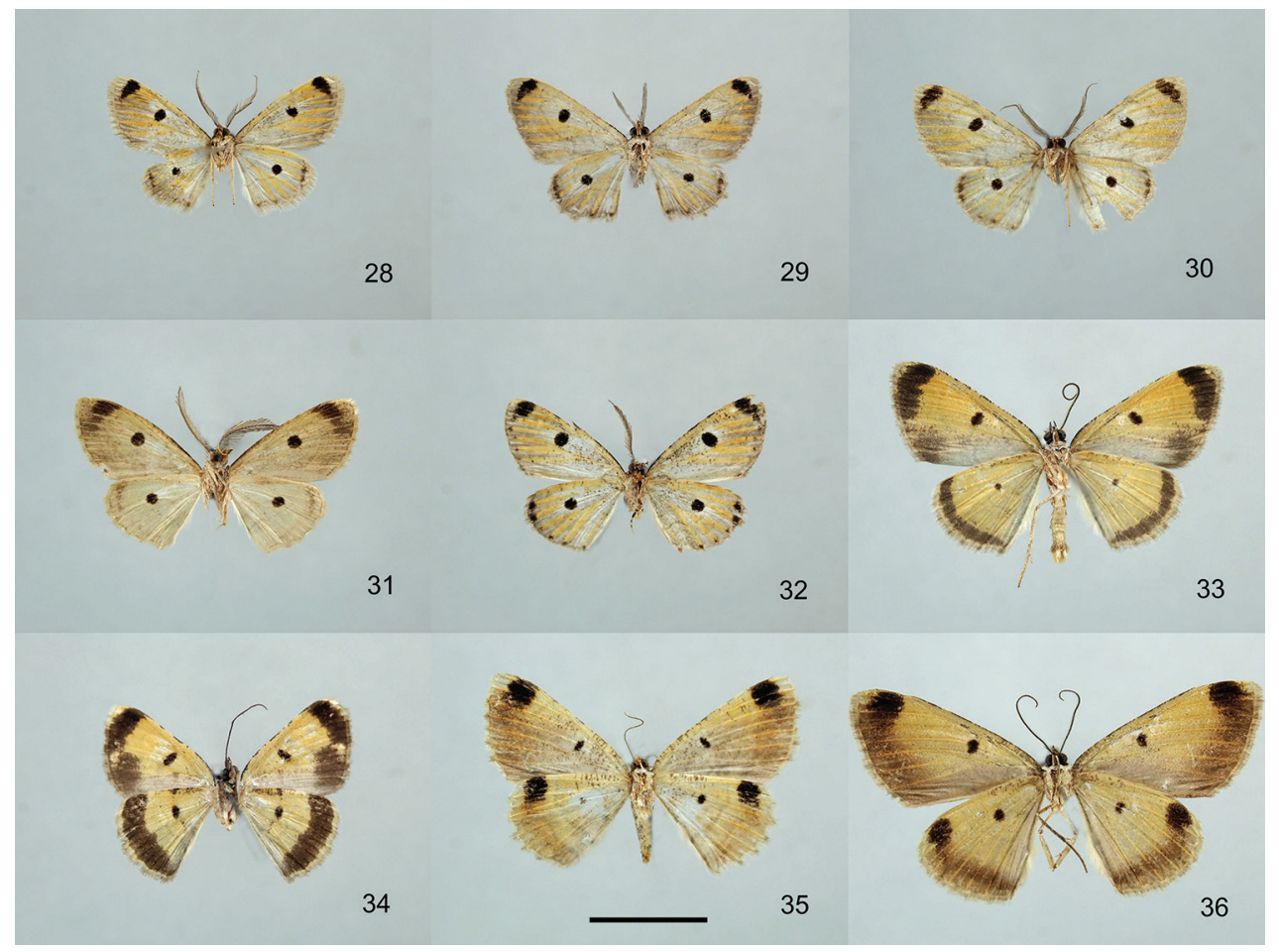

Figures 28-36. Specimens of the genera Orbamia, Rabomia, and Morabia, ventral view. 28 Orbamia abiyi 29 O. emanai 30 O. emanai lenzi 3 I O. pauperata 32 O. balensis 33 Rabomia subaurata $34 R$. obscurior 35 Morabia politzari 36 M. brunnea. Scale bars: $1 \mathrm{~cm}$.

Paratypes: $1{ }^{\lambda} 1$, , North Zambia, Mutinondo, 1390 m, wet Miombo, 29.XII.2010, $12^{\circ} 23^{\prime} 30^{\prime S}$, 31 ${ }^{\circ} 19^{\prime} 23^{\prime \prime E}$, light trap, J. Lenz legit (ZSM G 20913/ㅇ); 10․ S. Ethiopia SN, Arba Minch, below Hotel Bekele Molla, thornbush, $1310 \mathrm{~m}, 10^{\circ} 26^{\prime} \mathrm{N}, 39^{\circ} 53^{\prime} \mathrm{E}$, 29.IV.2008, leg. Hacker \& Schreier (ZSM G 20556); 1 ภे, Tanzania, Morogoro region, Mikesse Hills, 420 m, 06 $40.15^{\prime}$ 'S, 03757.57'E, 21.XII.2005, Ph. Darge; 1 우, Tanzania, Rukwa prov., Mbizi mts, entre Kisungu et Muze, 1415 m, 07 $43.82^{\prime} \mathrm{S}, 031^{\circ} 32.48^{\prime} \mathrm{E}$, 14.v.2004, leg. Ph. Darge; $10^{\top}$, Namibia, Kavango distr., $17^{\circ} 52^{\prime} \mathrm{N}, 19^{\circ} 39^{\prime} \mathrm{E}, 16 \mathrm{~km} \mathrm{~W}$ Rundu, (Okavango) Kavango river area, 28.II.2006, leg. H. Hacker \& H.P. Schreier (ZSM G 20932); 20̂, Rwanda, S.E. Rusumo, 1300 m, 29.XII.1975, leg. B. Turlin (ZSM G 13625; BC ZSM Lep ); 10, id., 25.3.1975 (ZSM).

Etymology. The name honours his Excellency Dr Abiy Ahmed Ethiopia’s Prime Minister, the 2019 Nobel Peace Prize Laureate, for his tremendous contributions to Ethiopia and the Horn of African geopolitics.

Differential features (COI sequences, photographs of adults and their genitalia see https://dx.doi.org/10.5883/DS-ORBAMIA): Adult: Forewing length: 10-12 mm. Upperside of wings: Ground colour pale grey with slight brown suffusion, pattern brown with very slight orange tinge. Underside: Ground colour whitish, orange be- 
tween veins, apical spots on forewing conspicuous, sharply bordered, on hindwing terminal fascia usually diffuse, in Tanzania hindwing terminal fascia uninterrupted. Male genitalia: Uncus narrow, digitiform, valva straight, broad, dorsal process with conspicuous, stout hook at tip, cornutus narrow and long $(2.2-2.3 \mathrm{~mm})$. Female genitalia: Lamella ante- and post-vaginalis fused, oval (length $0.75-0.85 \mathrm{~mm}$ ), signum weakly sclerotised, small, transverse ridge straight $(0.17-0.2 \mathrm{~mm})$.

Remarks. Populations from western Ethiopia with darker upperside of wings, on hindwing underside dark pattern reduced to apex. See also remarks to the following species.

\section{Orbamia emanai Hausmann \& Tujuba, sp. nov.} http://zoobank.org/5A4DFBCF-0D52-4378-AF90-84611329CBAE BIN: BOLD: ABW6858

Figures 11, 29, 47, 65, 79

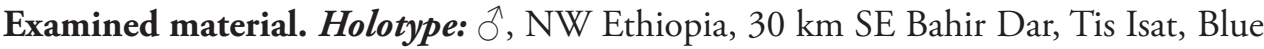
Nile falls, $1640 \mathrm{~m}, 11^{\circ} 29^{\prime} 08^{\prime \prime N}, 37^{\circ} 35^{\prime} 28^{\prime E}$, 25.VI.2008, leg. Hacker \& Schreier (ZSM G 20917).

Paratypes: $30{ }^{\wedge} 8$, NW Ethiopia, $30 \mathrm{~km}$ SE Bahir Dar, Tis Isat, Blue Nile falls, $1640 \mathrm{~m}, 1^{\circ} 29^{\prime} 08^{\prime \prime N}, 37^{\circ} 35^{\prime} 28^{\prime \prime E}, 25 . V I .2008$, leg. Hacker \& Schreier; $1 \delta^{\Uparrow}$, S. Ethiopia S.N., $12 \mathrm{~km} \mathrm{~W} \mathrm{Jinka,} \mathrm{border} \mathrm{of} \mathrm{Mago} \mathrm{N.P.,} 930 \mathrm{M}$ (lux), 05¹8'47"N, 3644'07"E, 6.V.2008, leg. Hacker \& Schreier (ZSM G 20910/; ZSM G 20916/ㅇ).

Additional material (exactly barcode-sharing): $8 \delta^{\wedge}$ 으, Botswana, Central distr. $15 \mathrm{~km}$ NW Francistown, river Tati, $1030 \mathrm{~m}, 21^{\circ} 02^{\prime} \mathrm{S}, 2^{\circ} 32^{\prime} \mathrm{E}, 19.1 \mathrm{I} .2006$, Hacker \&

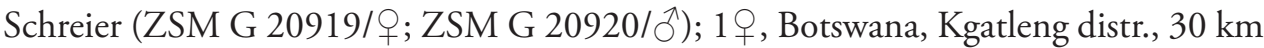
NNE Gaborone, 980 m (lux), 19.II.2006, leg Hacker \& Schreier; 2犬̂, Botswana, Central district $10 \mathrm{~km} \mathrm{SSE} \mathrm{Nata,} \mathrm{Sua} \mathrm{pan,} 930 \mathrm{~m}$ (lux) 2009'S, 26²6'E, 20.II.2006, leg Hacker \& Schreier.

Etymology. Named after Emana Getu, a senior professor of Entomology, at Addis Ababa University for his immense contributions to the field of entomology.

Differential features (COI sequences, photographs of adults and their genitalia see https://dx.doi.org/10.5883/DS-ORBAMIA): Adult: Forewing length: 9-12 mm. Upperside of wings: Ground colour comparatively dark. Underside: Ground colour pale grey, on hindwing with slight orange tinge, on forewing orange between veins, apical spots on forewing conspicuous, sharply bordered, on hindwing slightly darker at apex only. Male genitalia: Uncus narrow, digitiform, valva straight, broad, dorsal process with conspicuous, stout hook at tip, cornutus narrow and very long $(2.5-3.0 \mathrm{~mm})$. Female genitalia: Lamella ante- and post-vaginalis fused, oval (length $0.8-0.85 \mathrm{~mm}$ ), signum weakly sclerotised, small, transverse ridge straight $(0.17-0.2 \mathrm{~mm})$.

Remarks. Morphological differences to the previous species small, but the genetic divergence correlates with darker wing colour, and a few characters in male genitalia (longer cornutus). Distribution areas of both species overlapping. 

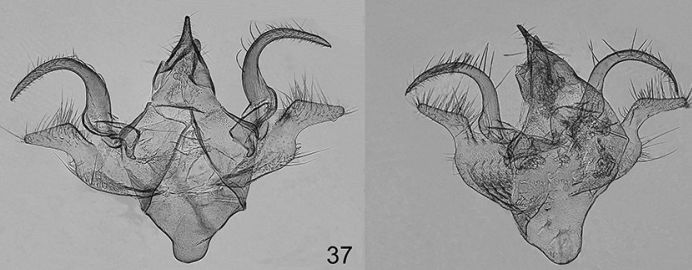

38

39

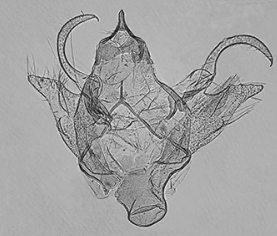

40

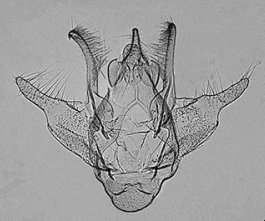

41
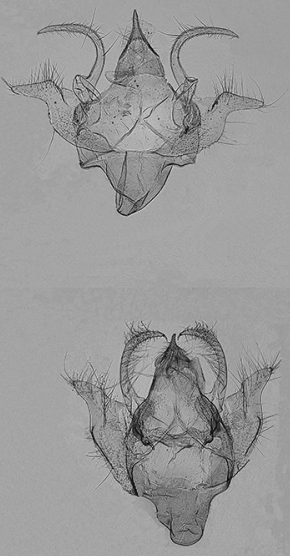

42

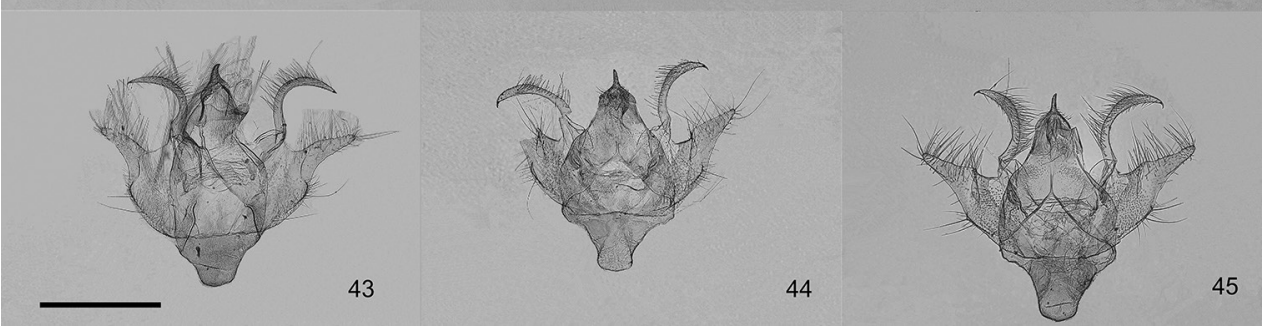

Figures 37-45. Male genitalia of the genus Orbamia. 37 Orbamia octomaculata 38 O. marginata 39 O. becki 40 O. renimacula 41 O. clarissima 42 O. clarior 43 O. obliqua 44 O. obliqua parva 45 O. ocellata. Scale bar: $1 \mathrm{~mm}$.

\section{Orbamia emanai lenzi Hausmann \& Tujuba, subsp. nov.}

http://zoobank.org/0FC250FF-B4AF-4461-8113-2D0CFEF85C5F BIN: BOLD: ABW6858

Figures 12, 30, 48, 66, 80

Examined material. Holotype: $\widehat{\jmath}$, Zambia, North Zambia, Mutinondo, 1390 m, wet Miombo, 01.I. 2011, 12²3'30"S, 31²19'23"E, light trap, J. Lenz legit, gen. prp. ZSM G 20917 (coll. ZSM G 20918).

Paratypes: 1 , Zambia, North Zambia, Mutinondo, $1390 \mathrm{~m}$, wet Miombo,

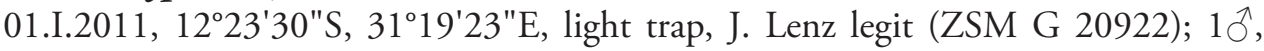
id., 29.XII.2010; 1ठ̄, Zambia, Northwest prov., Chiwona riverine forest, $1330 \mathrm{~m}$, -12.412S, 24.1910E, 12.IX.2015, leg Sàfiàn Szabolcs; $1 \sigma^{\Uparrow}$, S. Africa [sic!], S. Malawi, Nsanje distr. $25 \mathrm{~km} \mathrm{~S}$ Blantyre, Mwabvi reserve, $16^{\circ} 39.20^{\prime} \mathrm{S}, 35^{\circ} 03.03^{\prime} \mathrm{E}, 10 . X \mathrm{XI} .2010$, 127 m, Ustjuzhanin \& Kovtunovich (ZSM G 20907).

Etymology. Named after Jürgen Lenz, Harare - Leipzig, active and experienced researcher and collector of geometrids in Africa, mainly Zambia and Zimbabwe.

Differential features (COI sequences, photographs of adults and their genitalia see https://dx.doi.org/10.5883/DS-ORBAMIA): Adult: Forewing length: 10-11 mm. 


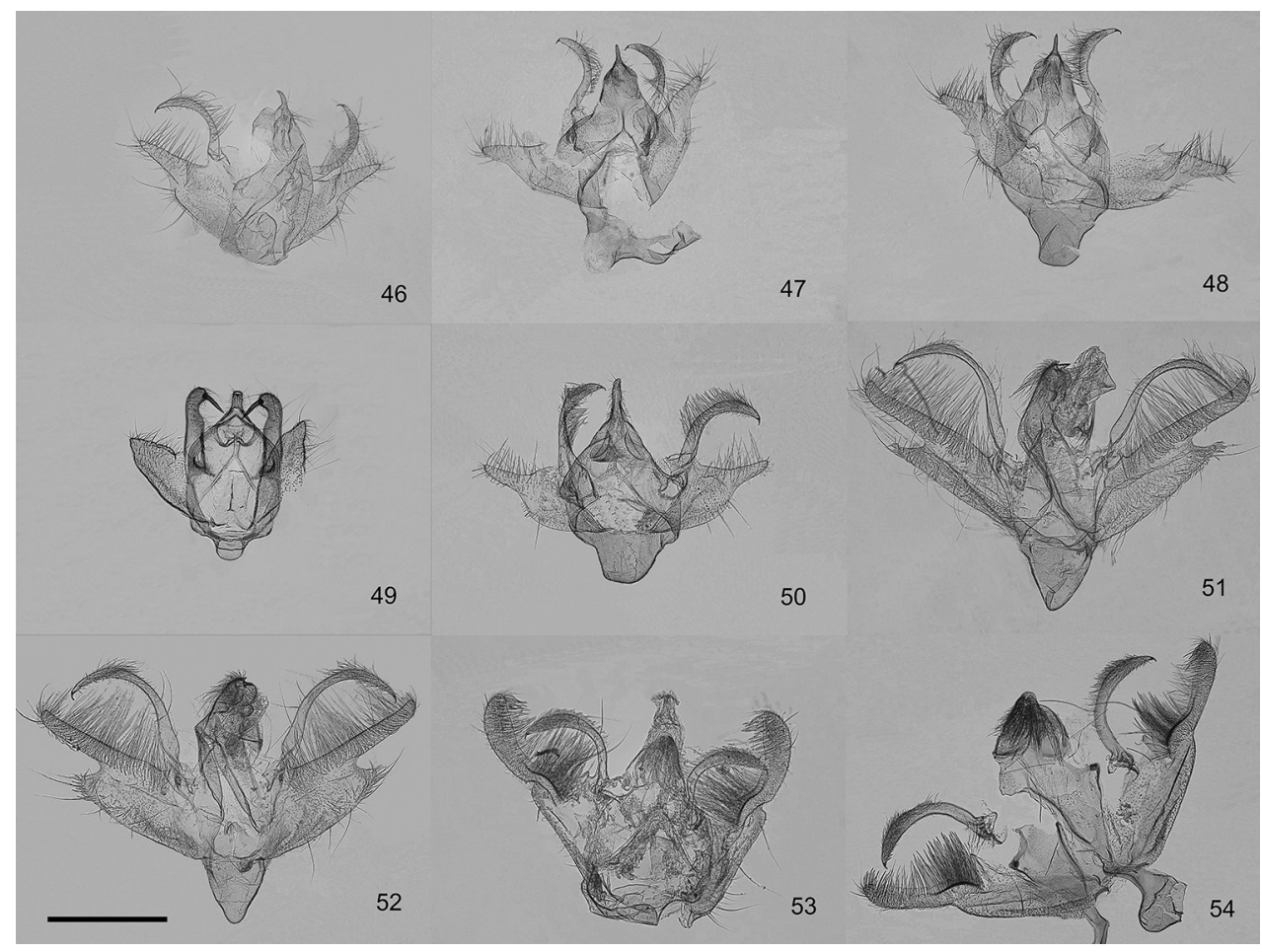

Figures 46-54. Male genitalia of the genera Orbamia, Rabomia, and Morabia. 46 Orbamia abiyi 47 O. emanai 48 O. emanai lenzi 49 O. pauperata 50 O. balensis 51 Rabomia subaurata 52 R. obscurior 53 Morabia politzari 54 M. brunnea. Scale bar: $1 \mathrm{~mm}$.

Upperside of wings: Ground colour much paler than in the nominotypical subspecies, very pale grey, with slight brown suffusion, mainly in the terminal area, pattern grey brown. Underside: Ground colour whitish beige, orange on veins, apical spots on forewing conspicuous, sharply bordered, on hindwing terminal fascia usually diffuse over more or less the whole termen. Male genitalia: Uncus narrow, digitiform, valva straight, broad, dorsal process with conspicuous, stout hook at tip, cornutus narrow and long $(2.7 \mathrm{~mm})$. Female genitalia: Lamella ante- and post-vaginalis fused, oval (length $0.75-0.85 \mathrm{~mm}$ ), signum weakly sclerotised, small, transverse ridge straight (0.17-0.2 mm).

Remarks. BIN-sharing but at $2.2 \%$ distance, much paler than the nominotypical subspecies, but little difference in genitalia.

\section{Orbamia pauperata Herbulot, 1966}

Figs 13, 31, 49, 67, 81

Orbamia pauperata: Herbulot (1966): 221 (Holotype $\$$ in ZSM: gen. prep. ZSM G 14466; locus typicus: Madagascar: Betioky, southern shore of Tsimanampetsotsa). 
Note. So far, without BIN, holotype with short barcode fragment (BC ZSM Lep 81698).

Examined material. $2 \lesssim 3$ q from Madagascar, including holotype (ZSM G 13619/q).

Differential features (COI sequences, photographs of adults and their genitalia see https://dx.doi.org/10.5883/DS-ORBAMIA): Adult: Forewing length: 10-12 mm. Upperside of wings: Ground colour pale grey. Underside: Ground colour beige, without yellow tinge, apical spot present on forewing. Male genitalia: Uncus digitiform, saccus shortly projecting, round, valva short, broad, tapered at tip, dorsal process stoutly sclerotised, with a conspicuous, very long spine at tip, aedeagus short $(1.2 \mathrm{~mm})$, cornutus very short $(0.7 \mathrm{~mm})$ and S-shaped at tip. Female genitalia: Lamella anteand post-vaginalis developed as two separate, narrow, transverse sclerites, posteriorly bilobed, signum rhomboid, transverse ridge straight $(0.25 \mathrm{~mm})$.

Remarks. Phylogenetically the most isolated species within this genus, based on large differences in morphology and genetics, the latter, however, just based on a short barcode fragment of the holotype.

\section{Orbamia balensis Hausmann \& Tujuba, sp. nov.}

http://zoobank.org/:0948AD35-4B39-4FF5-A210-5AAD56A1BE4C

BIN: BOLD: AEA2800

Figures 14, 32, 50, 68

Examined material. Holotype: $\widehat{\jmath}$, Ethiopia Oromia, Bale $8 \mathrm{~km} \mathrm{S.} \mathrm{Dolo} \mathrm{Mena,}$ 1200 m, IV.2017, leg. R. Beck, coll. ZSM (ZSM G 20914).

Paratypes: $2 \precsim 2$, Ethiopia Oromia, Bale 8 km S. Dolo Mena, 1200 m, IV.2017, leg. R. Beck (ZSM G 20948/) ); 1q, Äthiopien, prov. Oromia, Dolo Mena, 30 km, S., $1080 \mathrm{~m}$ (savanna) $06^{\circ} 13.53^{\prime} \mathrm{N}, 39^{\circ} 46.82^{\prime} \mathrm{E}, 14 . \mathrm{IV} .2019$, Robert Beck (ZSM).

Etymology. The name refers to the type locality in the Bale mountains.

Differential features (COI sequences, photographs of adults and their genitalia see https://dx.doi.org/10.5883/DS-ORBAMIA): Adult: Forewing length: 11-13 mm. Upperside of wings: Ground colour comparatively dark, with much dark brown suffusion, pattern not well contrasted. Underside: Ground colour beige, with pale orange tinge on veins, apical spots on forewing conspicuous, sharply bordered, on hindwing slightly darker at apex only, remnants of dotted terminal fascia on all wings. Male genitalia: Uncus stout, very long, saccus broad, valva straight, 'very broad at base, narrow at tip, dorsal process stoutly sclerotised, with conspicuous, stout hook at tip, cornutus narrow and short $(1.1 \mathrm{~mm})$. Female genitalia: Lamella ante- and post-vaginalis fused, heart-shaped, short (length $0.6 \mathrm{~mm})$, signum small, transverse ridge straight $(0.2 \mathrm{~mm})$.

Rabomia Hausmann \& Tujuba, gen. nov. http://zoobank.org/C6E24B77-0F95-457D-9034-26348A26F071

Type species. Ectropis? subaurata Warren, 1899. 


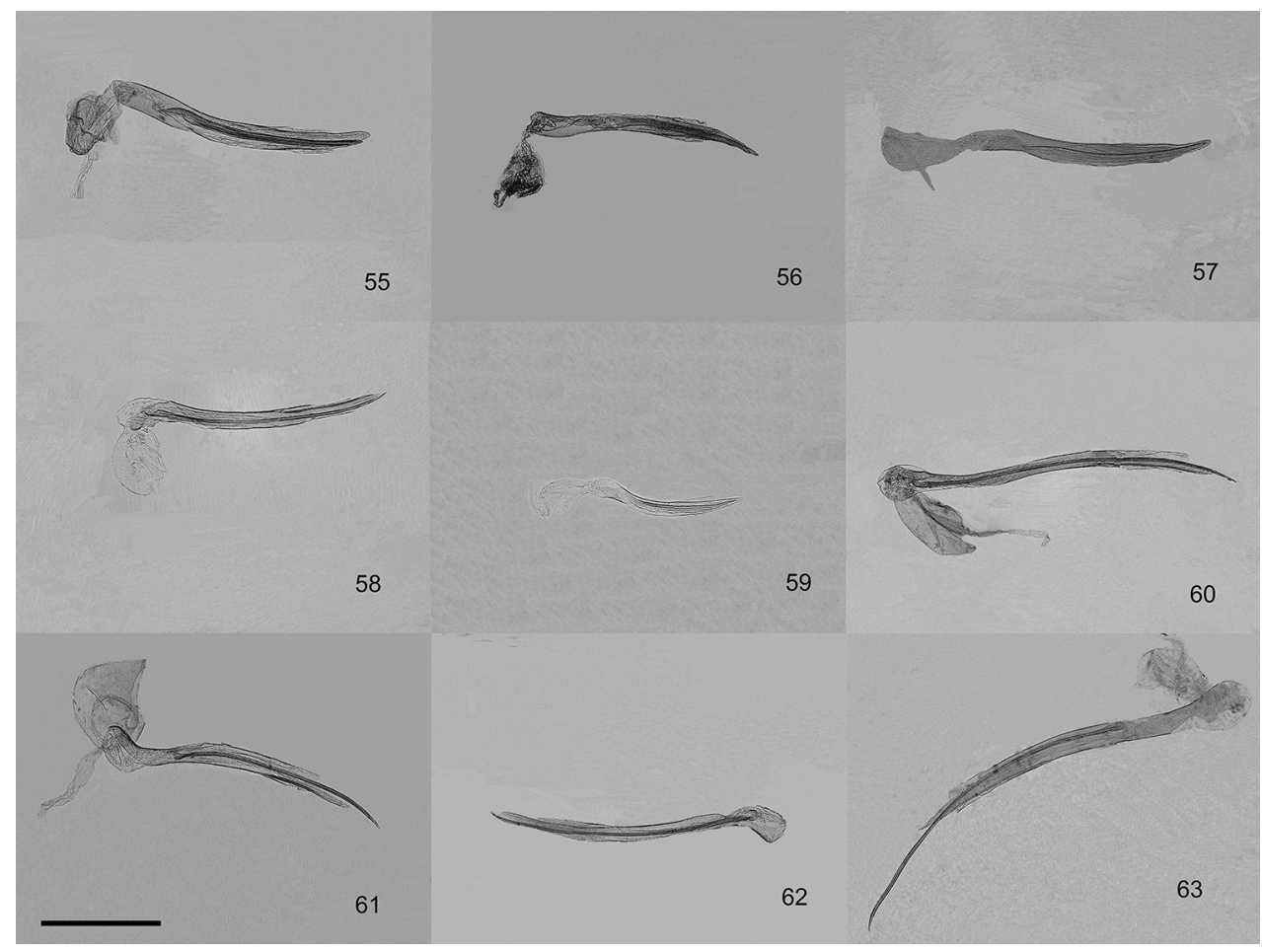

Figures 55-63. Aedeagus of male genitalia of the genus Orbamia. 55 Orbamia octomaculata 56 O. marginata 57 O. becki 58 O. renimacula 59 O. clarissima 60 O. clarior 61 O. obliqua 62 O. obliqua parva 63 O. ocellata. Scale bar: $1 \mathrm{~mm}$.

Etymology. The name is an anagram of the sister genus Orbamia, similarly to the anagram used by Herbulot (1966) when transforming the name Boarmia to Orbamia.

Differential features (COI sequences, photographs of adults and their genitalia see https://dx.doi.org/10.5883/DS-ORBAMIA). Adult: Male antennae ciliate-fasciculate, female antennae filiform. Palpi of both sexes broad, bushy scaled, length in male 1.0, in female 1.5 times diameter of eye. Hind tibia of both sexes with two pairs of unequal spurs. Upperside of wings with inconspicuous, elongate discal spots, postmedial line of forewings sharp, strongly curved. Underside yellow, black terminal fascia conspicuous. Male genitalia: Uncus with three short sclerotised spines, lateral lobes below uncus swollen, saccus triangular, dorsal process of valva naked, with curved spinule at tip, aedeagus comparatively broad, with bundle of small cornuti at base, vesica condensed to a broad cornutus-like sclerite at tip. Female genitalia: Lamellae ante- and post-vaginalis sclerotised (elongate rhomboid), posteriorly rounded, ductus bursae sclerotised, long, helicoid, longitudinally furrowed, corpus bursae membranous, signum large, star-shaped.

Genetic data and phylogeny. The maximum likelihood analysis of COI barcode data supports the monophyly of the genus Rabomia gen. nov. and sister group relationship with (Pycnostega+Dorsifulcrum) (cf. Table 1, Fig. 85). However, phylogenies at 
genus level need to be considered with caution, when they are inferred from COI data. More research is needed to investigate the potential (re-) assignment of Dorsifulcrum to the Cassymini after having been excluded from that tribe in Brehm et al. (2019).

Remarks. In some morphological features (e.g., female signum and ductus bursae) the genus Rabomia gen. nov. is transitional to the genus Dorsifulcrum Herbulot, 1979, but is genetically clustering separately, underside of wings with black fascia only in the terminal area.

Rabomia subaurata (Warren, 1899), comb. nov.

BIN: BOLD: AAM3217

Figures 15, 33, 51, 69, 82

Ectropis? subaurata: Warren (1899): 306 (Holotype \$ in NHMUK; locus typicus: [Zambia]: Mpeta, Loangwa River, off the Zambesi).

Examined material (ZSM). $10{ }^{\wedge} \uparrow$ from Zambia, Malawi, (southernmost) Democratic Republic of the Congo (Elisabethville [Lubumbashi]) (ZSM G 20923/ ${ }^{\text {; }}$ ZSM G20924 +).

Differential features (COI sequences, photographs of adults and their genitalia see https://dx.doi.org/10.5883/DS-ORBAMIA): Adult: Forewing length: 13-14 mm. Upperside of wings: Ground colour comparatively dark grey. Underside: Ground colour yellow, discal spots small, terminal fascia narrow at centre of forewing termen interrupted by a large yellow area. Male genitalia: Valva long and narrow, sacculus narrowly projecting at tip, at the base of aedeagus a bundle of eight comparatively long $(0.3 \mathrm{~mm})$ microcornuti. Female genitalia: Star-shaped signum large, diameter 0.7-0.9 mm.

\section{Rabomia obscurior Hausmann \& Tujuba, sp. nov.}

http://zoobank.org/73A152CB-ADB8-4B67-AC34-A3F1BB1E16A2

BIN: BOLD: ABV9564

Figures 16, 34, 52, 70, 83

Examined material. Holotype: $q$, Burkina Faso (Upper Volta), Bobo Dioulesso, 6.10.81, leg. Dr Politzar, coll. ZSM (G 20915).

Paratypes: 1웅 Burkina Faso (Upper Volta), Bobo Dioulesso, 13.11.85, leg. Dr

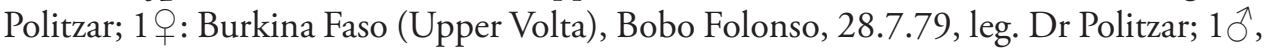
id., 10.11.74; 10, Nigeria, Kaduna, 3.vii.1970, leg. Politzar, coll. ZSM (G 20926); 1ㅇ, id., 28.vi.1970; 19, id., 26.vi.1970; 19, id., 8.vii.1970; 10, North Nigeria, Kogin Kano Game Reserve, 15.vi.1974, leg. Dr Politzar; 1엉 Cameroon, Yala Yarna, 40 km N of Ngaoundéré, 735 m, 22.vii.1974, leg. Gilles Clément (ZSM G 20925).

Etymology. The name refers to the darker colouration of wings (Lat. obscurior = darker). 


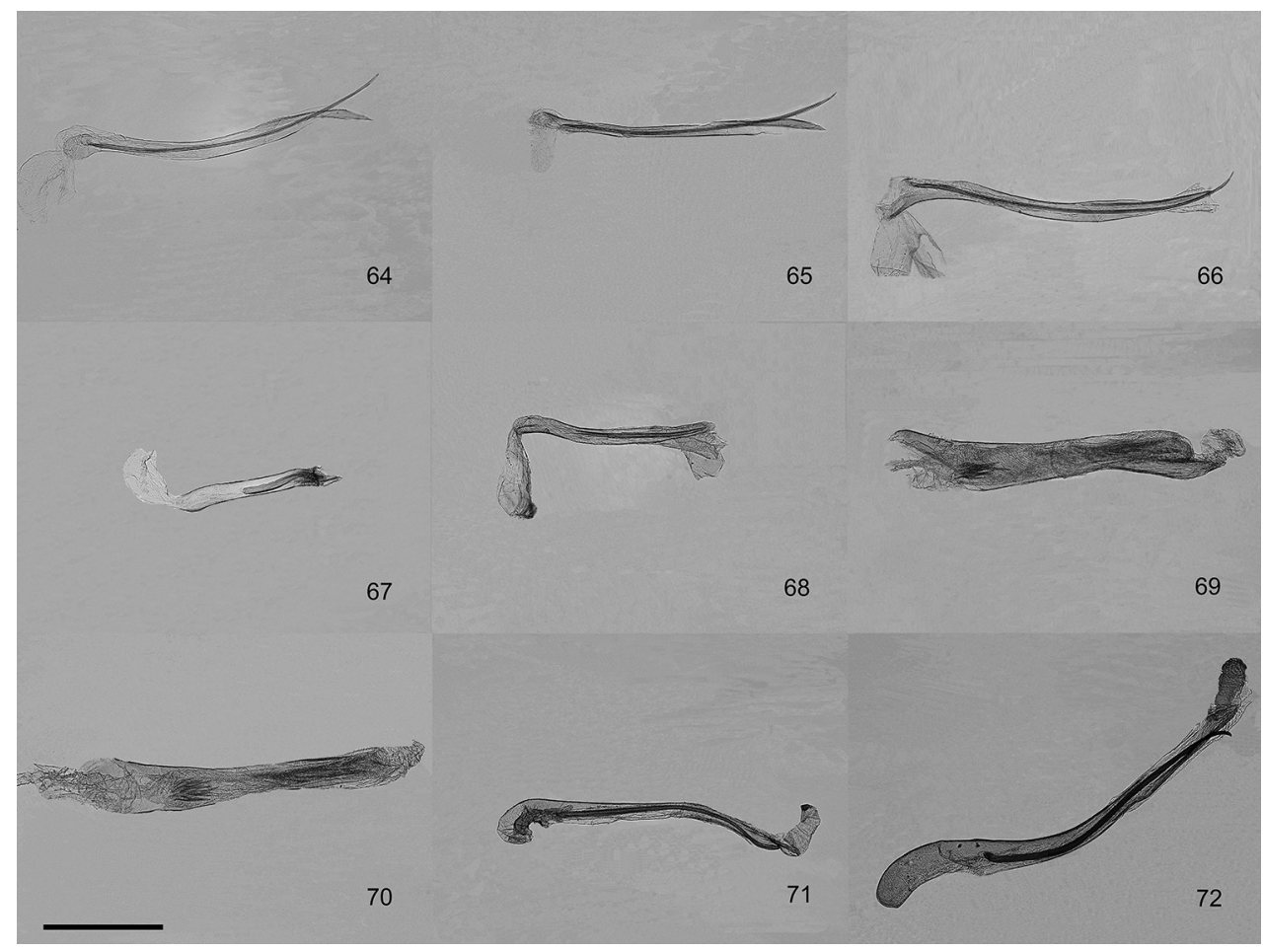

Figures 64-72. Aedeagus of male genitalia of the genera Orbamia, Rabomia, and Morabia. 64 Orbamia abiyi 65 O. emanai 66 O. emanai lenzi 67 O. pauperata 68 O. balensis 69 Rabomia subaurata 70 R. obscurior 71 Morabia politzari 72 M. brunnea. Scale bar: $1 \mathrm{~mm}$.

Differential features (COI sequences, photographs of adults and their genitalia see https://dx.doi.org/10.5883/DS-ORBAMIA): Adult: Forewing length: 12-14 mm. Upperside of wings: Ground colour with darker suffusion than in $R$. subaurata, medial fascia dark and conspicuous on all wings, sometimes anastomosing with postmedial line on forewing. Underside: Ground colour yellow, discal spots larger than in R. subaurata, terminal fascia much broader, at centre of forewing termen interrupted by a small yellow spot or even uninterrupted. Male genitalia: Valva long and narrow, sacculus edged at tip, only shortly projecting, at the base of aedeagus a bundle of twelve comparatively short $(0.15-$ $0.2 \mathrm{~mm}$ ) microcornuti. Female genitalia: Star-shaped signum large, diameter $0.8-1.0 \mathrm{~mm}$.

Morabia Hausmann \& Tujuba, gen. nov. http://zoobank.org/D7BB6DF9-B1CD-4D00-8A15-A6906DFB2B56

Type species. Morabia politzari Hausmann \& Tujuba, sp. nov.

Etymology. The name is an anagram of the sister genus Orbamia, similarly to the anagram used by Herbulot (1966) when transforming the name Boarmia to Orbamia. 


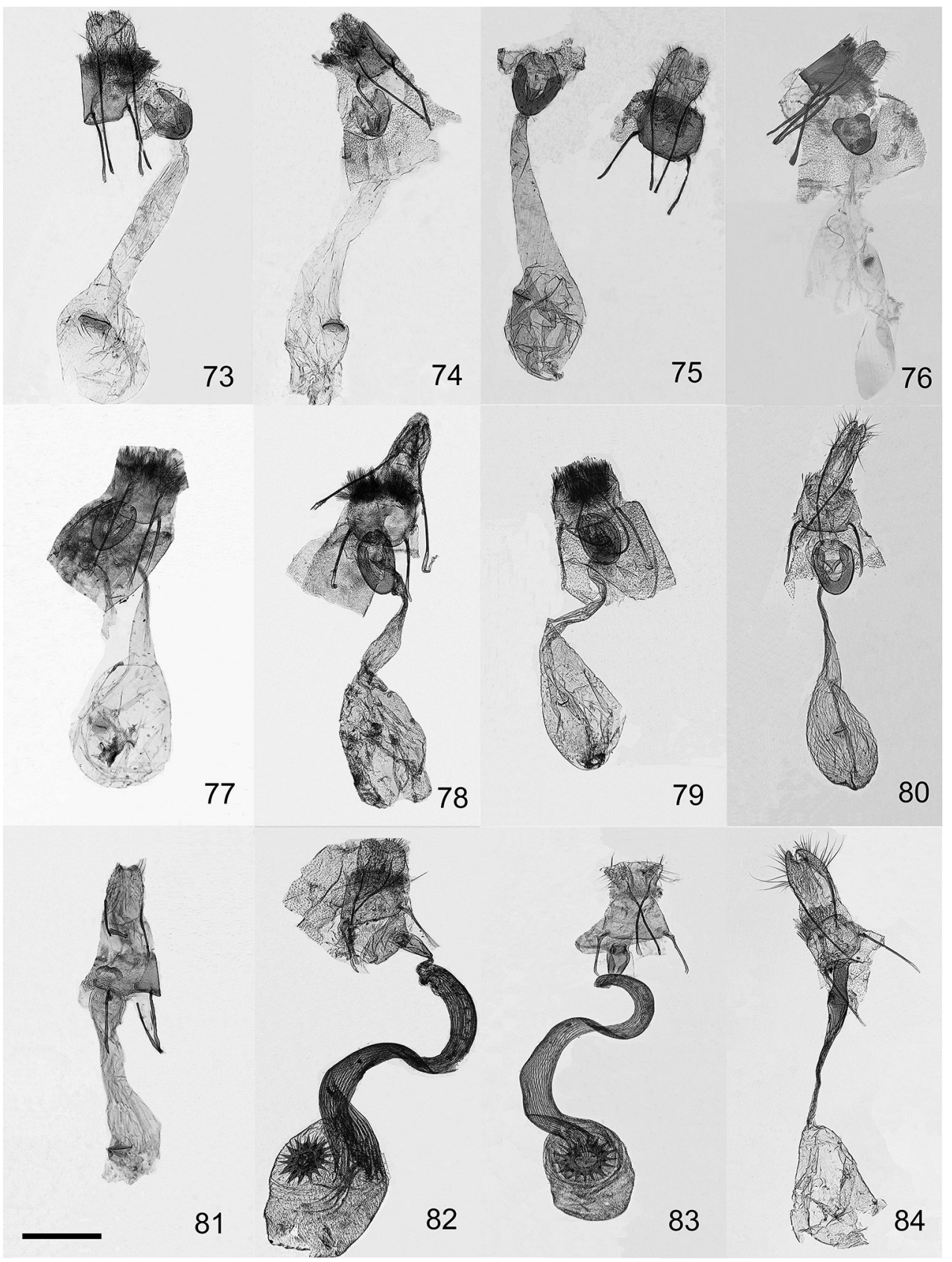

Figures 73-84. Female genitalia of the genera Orbamia, Rabomia, and Morabia. 73 Orbamia octomaculata 74 O. becki 75 O. renimacula 76 O. clarissima 77 O. ocellata 78 O. abiyi 79 O. emanai 80 O. emanai lenzi 8 I O. pauperata 82 Rabomia subaurata 83 R. obscurior 84 Morabia politzari. Scale bar: $1 \mathrm{~mm}$. 


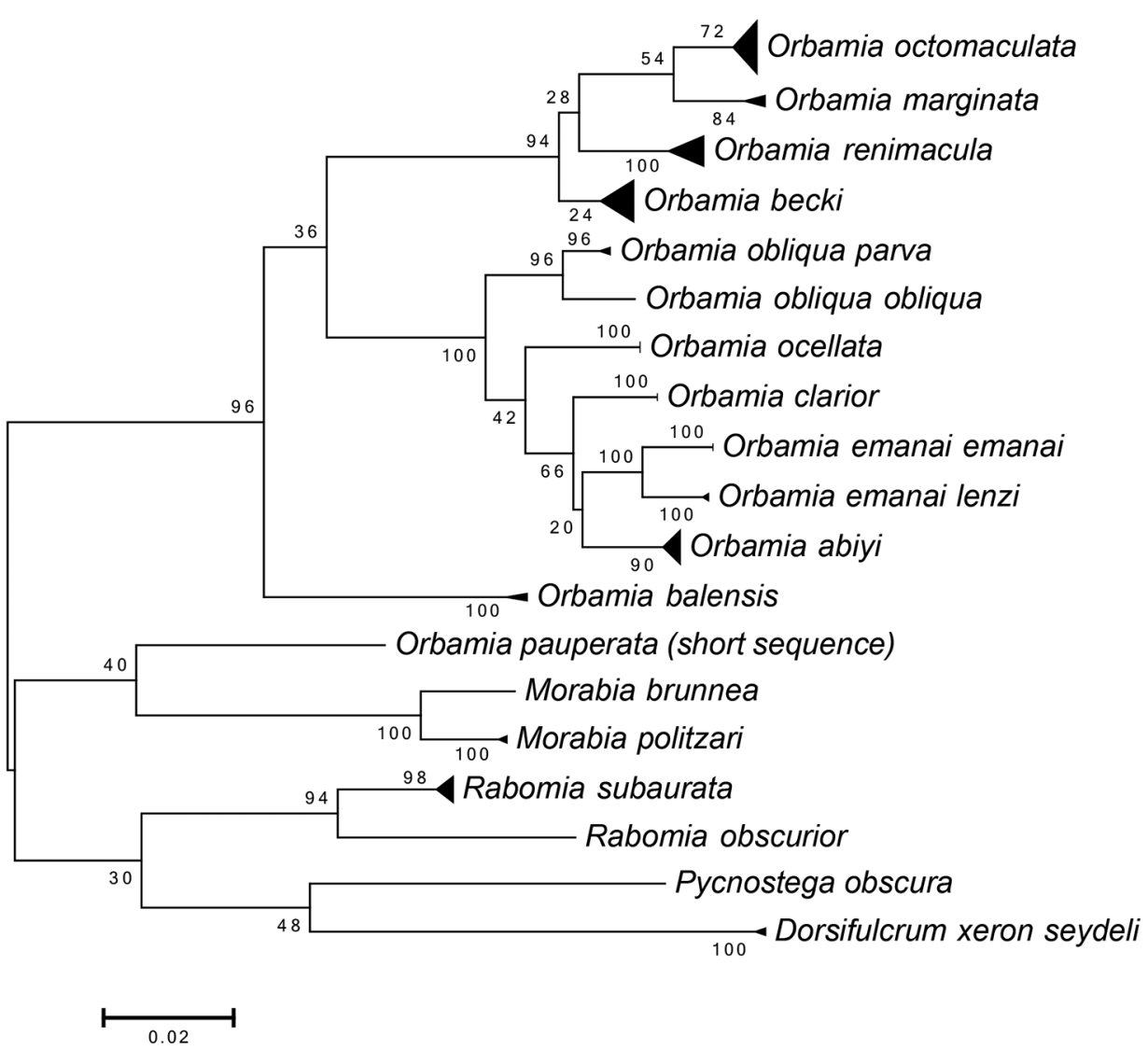

Figure 85. Maximum Likelihood Tree of COI data of the genera Orbamia, Rabomia, and Morabia, 50 Bootstrap Replications, Tamura-Nei model, uniform rates, built with Mega 6 software (Tamura et al. 2013; scale bar $=2 \%$ ), for original data see https://dx.doi.org/10.5883/DS-ORBAMIA.

Differential features (COI sequences, photographs of adults and their genitalia see https://dx.doi.org/10.5883/DS-ORBAMIA). Adult: Male antennae ciliate-fasciculate, female antennae filiform. Palpi of both sexes broad, bushy scaled, length 1.0-1.5 times diameter of eye. Hind tibia of both sexes with two pairs of unequal spurs. Upperside of wings with discal spots vestigial, medial line zigzagging, terminal line conspicuous, zigzagging on all wings. Underside beige, with a few yellowish scales and a sharp black spot in forewing apex. Male genitalia: Uncus very short, rounded saccus shallowly projecting, dorsal process of valva strongly setose, valva long and narrow, curved, strongly setose, mainly at centre, aedeagus with long and stout cornutus. Female genitalia: Apophyses long and fine. Lamellae ante- and post-vaginalis membranous, ductus bursae straight, anteriorly membranous, posteriorly dilated and towards antrum strongly sclerotised, corpus bursae membranous, pyriform, signum absent. 
Genetic data and phylogeny. The maximum likelihood analysis of COI barcode data suggests the monophyly of the genus Morabia gen. nov. and an isolated position from (Rabomia gen. nov. (Pycnostega+Dorsifulcrum)) and from genus Orbamia (cf. Table 1, Fig. 85). However, phylogenies at genus level need to be considered with caution, when they are inferred from COI data.

\section{Morabia politzari Hausmann \& Tujuba, sp. nov.}

http://zoobank.org/38C88B00-2394-4C29-81A9-9E58E06B058E

BIN: BOLD: ABX0432

Figures $17,35,53,71,84$

Examined material. Holotype: $\odot$, Kenya, Sokoke Forest, 31.7.94, leg. Dr. Politzar, coll. ZSM.

Paratypes: 10, Kenya, Watama, 2.viii.1973, leg. Politzar, coll. ZSM (G 20934); 1오, id., 30.vii.1973; 1으, Kenya, Sokoke Forest, 31. Vii. 1994, leg. Politzar; 1 , id, 2.vii.1994; 3우 Tanzanie Bagamoyo dist. Vigwaza, $231 \mathrm{~m}, 06^{\circ} 42.89^{\prime} \mathrm{S}, 038^{\circ} 52.52^{\prime} \mathrm{E}$, 30.III 2014, leg. Ph. Darge; 1 우, Tanzanie, Pwani region, Ruvu forest reserve, $220 \mathrm{~m}$, 06 $57.27^{\prime}$ S, 038 $49.36^{\prime}$ E, 03.III.2014, leg. Ph. Darge (ZSM); 1ㅇ, Coll. Mus. Tervuren, E.ville [DR Congo], 16.X.1955, Seydel (ZSM G 20935).

Etymology. The name refers to Dr. Heinz Politzar (1938-2007) for his great merits in collecting and studying African Lepidoptera (see Hacker and Hausmann 2010).

Differential features (COI sequences, photographs of adults and their genitalia see https://dx.doi.org/10.5883/DS-ORBAMIA): Adult: Forewing length: 12$14.5 \mathrm{~mm}$. Upperside of wings: Ground colour whitish beige. Underside: Ground beige, with slight yellow or orange tinge, mainly on forewing, discal spots small, both wings with sharp, black apical spot. Male genitalia: Uncus very short, rounded, saccus shallowly projecting, dorsal process of valva strongly setose, valva long and narrow, curved, strongly setose, mainly at centre, aedeagus (length $2.5 \mathrm{~mm}$ ) with long and stout cornutus $(1.9 \mathrm{~mm})$, sigmoid at tip. Female genitalia: Lamellae ante- and postvaginalis membranous, ductus bursae straight, anteriorly membranous, posteriorly dilated and towards antrum strongly sclerotised, corpus bursae membranous, pyriform, signum absent.

\section{Morabia brunnea Hausmann \& Tujuba, sp. nov.}

http://zoobank.org/E25394ED-1400-454C-BDC5-882DAB89179C

BIN: BOLD: ABW6916

Figures 18, 36, 54, 72

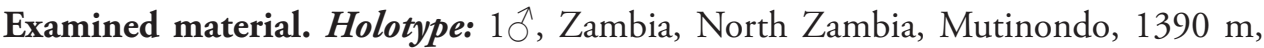
wet Miombo, 27.XII. 2010, $12^{\circ} 23^{\prime} 30^{\prime \prime S}, 31^{\circ} 19^{\prime} 23 " \mathrm{E}$, light trap, J. Lenz legit, coll. ZSM (G 20945). 
Etymology. The name refers to the unusual, brownish ground colour of the wings (Lat. brunneus, $-\mathrm{a}$, $\mathrm{um}=$ brown).

Differential features (COI sequences, photographs of adults and their genitalia see https://dx.doi.org/10.5883/DS-ORBAMIA): Adult: Forewing length: $17.5 \mathrm{~mm}$. Upperside of wings: Ground colour warm brown, pattern as in $M$. politzari. Underside: Ground pale brownish yellow, discal spots conspicuous, both wings with sharp, black apical spot and diffuse brown terminal fascia. Male genitalia: Uncus very short, rounded, strongly setose, saccus shallowly projecting, dorsal process of valva strongly setose, valva long and narrow, curved, strongly setose, mainly at centre, aedeagus longer $(3.5 \mathrm{~mm})$ than in the preceding species, S-shaped, with stout, S-shaped cornutus, longer than in the preceding species $(2.3 \mathrm{~mm})$. Female genitalia unknown.

\section{Acknowledgements}

The genetic analyses have received support from Paul DN Hebert and the Biodiversity Institute of Ontario (BIO) and the Canadian Centre for DNA Barcoding (CCDB University of Guelph). The data management and analysis system BOLD was provided by Sujeevan Ratnasingham.

\section{References}

Banki O, Hobern D, Döring M, Remsen D (2019) Catalogue of Life Plus: A collaborative project to complete the checklist of the world's species. Biodiversity Information Science and Standards 3: e37652. https://doi.org/10.3897/biss.3.37652

Brehm G (2015) Three new species of Hagnagora Druce, 1885 (Lepidoptera, Geometridae, Larentiinae) from Ecuador and Costa Rica and a concise revision of the genus. ZooKeys 537: 131-156. https://doi.org/10.3897/zookeys.537.6090

Brehm G (2018) Revision of the genus Callipia Guenée, 1858 (Lepidoptera, Geometridae), with the description of 15 new taxa. European Journal of Taxonomy 404: 1-54. https:// doi.org/10.5852/ejt.2018.404

Brehm G, Murillo-Ramos L, Sihvonen P, Hausmann A, Schmidt BC, Ounap E, Moser A, Mörtter R, Bolt D, Bodner F, Lindt A, Parra LE, Wahlberg N (2019) New World geometrid moths (Lepidoptera: Geometridae): Molecular phylogeny, biogeography, taxonomic updates and description of 11 new tribes. Arthropod Systematics \& Phylogeny 77(3): 457-486.

De Carvalho MR, Bockmann FA, Amorim DS, Brandao CRF, de Vivo M, de Figueiredo JL, Britski HA, de Pinna MCC, Menezes NA, Marques FPL, Papavero N, Cancello EM, Crisci JV, McEachran JD, Schelly RC, Lundberg JG, Gill AC, Britz R, Wheeler QD, Stiassny MLJ, Parenti LR, Page LM, Wheeler WC, Faivovich J, Vari RP, Grande L, Humphries CJ, DeSalle R, Ebach MC, Nelson GJ (2007) Taxonomic impediment or impediment to taxonomy? A commentary on systematics and the cybertaxonomic-automation paradigm. Evolutionary Biology 34(3-4): 140-143. https://doi.org/10.1007/s11692-007-9011-6 
Forum Herbulot (2014) Forum Herbulot 2014 statement on accelerated biodiversity assessment statement (Community Consensus Position). In: Hausmann A (Ed.) Proceedings of the eighth Forum Herbulot 2014. How to accelerate the inventory of biodiversity (Schlettau, 30 June -4 July 2014). Spixiana 37(2): 241-242.

Hacker H, Hausmann A (2010) Obituary to Dr. Karlheinz Politzar. Esperiana Memoir 5: 35-37.

Hardwick DF (1950) Preparation of slide mounts of lepidopterous genitalia. Canadian Entomologist 82: 231-235. https://doi.org/10.4039/Ent82231-11

Hausmann A (2003) Die afrotropischen Arten der Gattung Hypochrosis Guenée, [1858] mit Beschreibung einer neuen Gattung (Cherbulois gen. nov.) und 17 neuer Arten (Lepidoptera, Geometridae, Ennominae). Spixiana 26(2): 97-128.

Hausmann A (2006) The geometrid moths of Yemen - With 50 new records for the country and description of 20 new taxa (Lepidoptera: Geometridae). Esperiana 12: 9-62, 10 colour plates.

Hausmann A, Parisi F, Sciarretta A (2016) The Geometrinae of Ethiopia II: Tribus Hemistolini, genus Prasinocyma (Lepidoptera: Geometridae, Geometrinae). Zootaxa 4065(1): 1-63. https://doi.org/10.11646/zootaxa.4065.1.1

Herbulot C (1966) Nouveaux Geometridae du Sud-ouest de Madagascar. Bulletin Mensuel Société Linnéenne de Lyon 35: 216-221.

Ivanova NV, Dewaard JR, Hebert PDN (2006) An inexpensive, automation- friendly protocol for recovering high-quality DNA. Molecular Ecology Notes 6: 998-1002. https://doi. org/10.1111/j.1471-8286.2006.01428.x

Kimura M (1980) A simple method for estimating evolutionary rates of base substitutions through comparative studies of nucleotide sequences. Journal of Molecular and Evolution.16(2): 111-120. https://doi.org/10.1007/BF01731581

Löbel H, Hausmann A (2019) "Geometridae mundi", a new way of summarizing the diversity of global Geometrid species. In: Hausmann A (Ed.) Proceedings of the tenth Forum Herbulot 2018. Integrative taxonomy, a multidisciplinary approach to answer complicated taxonomic questions (Stuttgart, 11-16 June 2018). Spixiana 42(2): 291-320.

Meierotto S, Sharkey MJ, Janzen DH, Hallwachs W, Hebert PDN, Chapman EG, Smith MA (2019) A revolutionary protocol to describe understudied hyperdiverse taxa and overcome the taxonomic impediment. Deutsche Entomologische Zeitschrift 66(2): 119-145. https://doi.org/10.3897/dez.66.34683

Murillo-Ramos LdC, Brehm G, Sihvonen P, Hausmann A, Holm S, Ghanavi HR, Ónap E, Truuverk A, Staude H, Friedrich E, Tammaru T, Wahlberg N (2019) A comprehensive molecular phylogeny of Geometridae (Lepidoptera) with a focus on enigmatic small subfamilies. PeerJournal 7: e7386. https://doi.org/10.7717/peerj.7386

Prout LB (1926) New Geometridae in the Tring Museum. Novitates Zoologicae 33: 179-188. https://doi.org/10.5962/bhl.part.21141

Ratnasingham S, Hebert PDN (2007) BOLD: the barcode of life data systems. Molecular Ecology Notes 7: 355-364. https://doi.org/10.1111/j.1471-8286.2007.01678.x

Riedel A, Sagata K, Suhardjono YR, Tänzler R, Balke M (2013a) Integrative taxonomy on the fast track - towards more sustainability in biodiversity research. Frontiers in Zoology 10(15). https://doi.org/10.1186/1742-9994-10-15 
Riedel A, Sagata K, Surbakti S, Tänzler R, Balke M (2013b) One hundred and one new species of Trigonopterus weevils from New Guinea. ZooKeys 280: 1-150. https://doi.org/10.3897/ zookeys.280.3906

Scoble MJ (1999) Geometrid Moths of the World: A Catalogue. CSIRO Publishing, Collingwood, Stenstrup, 1016 pp. https://doi.org/10.1071/9780643101050

Scoble MJ, Hausmann A (2007) Online list of valid and available names of the Geometridae of the world. http://www.lepbarcoding.org/geometridae/species_checklists.php

Tamura K, Stecher G, Peterson D, Filipski A, Kumar S (2013) MEGA6: Molecular Evolutionary Genetics Analysis Version 6.0. Molecular Biology and Evolution 30: 2725-2729. https://doi.org/10.1093/molbev/mst197

Tujuba TF, Sciarretta A, Hausmann A, Abate GA (2019) Lepidopteran biodiversity of Ethiopia: current knowledge and future perspectives. ZooKeys 882: 87-125. https://doi. org/10.3897/zookeys.882.36634

Van Nieukerken EJ, Kaila L, Kitching IJ, Kristensen NP, Lees DC, Minet J, Mitter C, Mutanen M, Regier JC, Simonsen TJ, Wahlberg N, Yen S, Zahiri R, Adamski D, Baixeras J, Bartsch D, Bengtsson BÅ, Brown JW, Bucheli SR, Davis DR, Prins J de, De Prins W, Epstein ME, Gentili-Poole P, Gielis C, Hättenschwiler P, Hausmann A, Holloway JD, Kallies A, Karsholt O, Kawahara AY, Koster J, Kozlov M, Lafontaine JD, Lamas G, Landry J, Lee S, Nuss M, Park K, Penz C, Rota J, Schintlmeister A, Schmidt BC, Sohn J, Solis MA, Tarmann GM, Warren AD, Weller S, Yakovlev RV, Zolotuhin VV, Zwick A (2011) Order Lepidoptera Linnaeus, 1758. In: Zhang Z-Q (Ed.) Animal biodiversity: an outline of higher-level classification and survey of taxonomic richness. Zootaxa 3148: 212-221. https://doi.org/10.11646/zootaxa.3148.1.41

Wallengren HDJ (1872) Bidrag till Södra Afrikas Fjärilfauna. Öfversigt af Kongliga Vetenskaps-Akademiens Förhandlingar 29(3): 41-61.

Warren W (1899) New Drepanulidae, Thyrididae, and Geometridae from the Aethiopian region. Novitates Zoologicae 6(3): 287-312. https://doi.org/10.5962/bhl.part.22168

Wheeler QD (2008) Taxonomic shock and awe. In: Wheeler QD (Ed.) The New Taxonomy. 76. CRC Press, Boca Raton, 211-226. https://doi.org/10.1201/9781420008562.ch10 